\begin{tabular}{|c|c|c|}
\hline $\begin{array}{l}\text { PKS } \\
\text { PUBLIC } \\
\text { KNOWLEDGE } \\
\text { PROJECT }\end{array}$ & $\begin{array}{c}\text { REVISTA DE GEOGRAFIA } \\
\text { (RECIFE) } \\
\text { http://ww.revista.ufpe.br/revistageografia }\end{array}$ & $\begin{array}{l}\text { OJS } \\
\frac{\text { OPEN }}{\text { OPENAL }} \\
\text { SYSTEMS }\end{array}$ \\
\hline
\end{tabular}

\title{
MODELO RAMPA: ESBOÇO METODOLÓGICO PARA DIMENSIONAMENTO DE VERTENTES
}

\author{
Rodrigo de Freitas Amorim¹, Fernando Moreira da Silva², Antonio Carlos de Barros Corrêa ${ }^{3}$
}

${ }^{1}$ Professor do Departamento de Geografia da Universidade Federal do Rio Grande do Norte. Email: rodrigofba@yahoo.com.br

2 Professor do Departamento de Geografia da Universidade Federal do Rio Grande do Norte. Email: fmoreira@ufrnet.com

${ }^{3}$ Professor do Departamento de Ciências Geográficas da Universidade Federal de Pernambuco. Email: dbiase2001@terra.com.br

Artigo recebido em 26/04/2017 e aceito em 22/08/2017

\begin{abstract}
RESUMO
A erosão do solo é um dos processos de degradação mais importante em ecossistemas, que decorre, também, do movimento das águas ao longo de uma vertente. As características da vertente influenciam diretamente na maior ou menor suscetibilidade à perda de solo. A mensuração do comprimento de vertente é um passo fundamental nos estudos de perda de solo e planejamento de práticas conservacionistas. Desta forma, o objetivo é propor um modelo analítico para o cálculo do comprimento de vertentes (rampa). Como metodologia fez-se uso dos teoremas de limite e derivada, com implementação na linguagem de programação "Linguagem Espacial para Geoprocessamento Algébrico" (LEGAL) disponível no software SPRING. Como protótipo fez-se uma aplicação na Microbacia Riacho Passagem, região semiárida do Rio Grande do Norte, e obteve-se um mapa com o comprimento de rampa, sendo parcialmente validado com visitas a campo. O modelo proposto possibilita melhorar de modo significativo os estudos voltados à compreensão da erosão do solo, uma vez que permite calcular os comprimentos de vertentes a partir dos valores de uma grade numérica de terreno.
\end{abstract}

Palavras-chave: Rampa, Vertente, Derivada, Erosão .

\section{SLOPE MODEL: AN OUTLINE METHODOLOGY FOR DESIGN OF SLOPES}

\begin{abstract}
Soil erosion is one of the degradation processes most important in ecosystems, which follows, also, the movement of water along a slope. The characteristics of the slope directly influence the susceptibility to soil loss. The measurement of the length of slope is a key step in studies of soil loss and planning of soil conservation practices. Thus, the goal is to propose an analytical model for calculating the length of slopes. The methodology chose to use the theorems of limit and derivative, with implementation with the Java programming language for GIS Algebraic Space (LEGAL) available in the software SPRING. As a result there was an application in Microbacia Riacho Passagem, semi-arid region of Rio Grande do Norte, and obtained a map of the length of the slope, and partially validated with field trips. The proposed model makes it possible to improve significantly the studies focused on soil erosion as it allows you to calculate the lengths of slopes from the resolution of a satellite image.
\end{abstract}

Key-words: Slope, Strand, Derived, Erosion. 


\section{INTRODUÇÃO}

O escoamento superficial da água na vertente, ocasionando erosão, é sem dúvidas o mais importante processo geomorfológico na esculturação da superfície terrestre, alterando as formas de relevo e influenciando na pedogênese dos solos ao longo do tempo. Por conseguinte, esse processo conduz a dinâmica de remodelagem constante da paisagem, dentro dos padrões morfoclimáticos de cada região.

O transporte de sedimento inicia-se quando o volume de água precipitada excede a capacidade de campo do solo, ocasionando o movimento de água vertente a baixo, enxurrada que vai aumentando de volume e velocidade à medida que se aproxima do seu destino final, um rio, riacho ou outro corpo hídrico que forma o nível de base local. Vale salientar que, o arraste do material que recobre as rochas só se desencadeia no momento em que a resistência de agregação das partículas do solo rompe e o sedimento parra ser transportado em suspensão, diluição, arraste ou saltitação.

O trabalho realizado pela enxurrada faz parte do ciclo hidrológico, tendo como motor propulsor a energia solar incidente na terra. Dentre outras variáveis, a erosão causada pelo escoamento superficial da água, no estado líquido, depende também do balanço hídrico e radiação solar incidente, variando conforme e localização geográfica do globo. Essa dinâmica cria e recria as paisagens, da mesma forma que interfere nos tipos de uso do solo. A paisagem, portanto, é o resultado de uma dinâmica instável da interação de elementos físicos, biológicos e antrópicos (BERTRAND, 1972).

O entendimento e análise do processo erosivo são de grande relevância, uma vez que sua ocorrência, tanto pela água como pelo vento, é responsável por grande parte da degradação dos solos no mundo. "A erosão foi um dos fatores mais importantes que causaram a queda das primeiras civilizações e impérios, cujas cidades arruinadas estão agora como despojos estéreis das terras mais férteis do mundo" (BERTONI \& LOMBARDI NETO 1999).

A erosão do solo é um processo natural e seu início remonta ao pré-cambriano, ou seja, o início da história geológica do planeta, não sendo de exclusividade do atual período em que os humanos exploram os recursos naturais. É importante salientar que a erosão do solo não é um processo que só ocorre quando das ações humanas, o que deixa transparecer a maioria dos trabalhos na área ambiental, os quais não consideram a dinâmica natural da paisagem. Na realidade, o que é preciso considerar, tem-se a ação antrópica proporcionando a aceleração do processo erosivo. Ou seja, taxas de erosão do solo superiores ao volume reposto 
pelos processos de intemperismo e pedogênese, produzindo uma diminuição do perfil do manto de intemperismo.

A erosão acelerada dos mantos de intemperismos, tanto no Brasil quanto no mundo, é resultantes da combinação do processo de uso desordenado do solo e da falta de políticas voltadas para sua conservação. A forma de exploração dos recursos naturais sempre ocorre sem que seja levada em consideração a capacidade de suporte ambiental, também conhecida como sensitividade da paisagem. No contexto da geomorfologia, na maioria dos casos de degradação decorre do binômio: delgados mantos de intempéricos e condições climáticas adversas, especialmente semiárida. Essa combinação junto com o manejo inadequado do solo vem ocasionando a perda de milhares de toneladas de solo todo ano.

No semiárido do Nordeste do Brasil, o fato mais marcante decorrente da erosão do solo é o processo de desertificação, especialmente no compartimento geomorfológico da Depressão Sertaneja, onde predomina o embasamento geológico cristalino, onde as condições naturais favorecem a denudação e a baixa pedogênese, diminuindo a capacidade de armazenamento de água e elevando a temperatura do perfil de intemperismo. Como consequência, tem-se a perda de espécies vegetais mais vulneráveis ao déficit hídrico e maiores temperaturas.

Nesse contexto, verifica-se uma intensa busca por modelos capazes de mensurar perdas de solo, de modo a avaliar os níveis de impacto da sociedade frente aos diferentes contextos agroambientais. As tentativas de desenvolver um método capaz de mensurar essas perdas em campo, em uma determinada área, começou no ano de 1940, na Região de Corn Belt, nos Estados Unidos, sendo que o modelo final só foi concluído em 1965 com as contribuições de Wischmeier e Smith (1978), com a proposta do modelo denominado de Equação Universal de Perda de Solos (EUPS).

Reconhecido como uma ferramenta importante para o estudo da erosão laminar, representando a perda de solo por unidade/área, em toneladas/ha/ano, o modelo da EUPS congrega fatores de ordem natural (erosividade da chuva, erodibilidade do solo, comprimento e declividade de rampa) e fatores de ordem antrópica (uso e manejo do solo e práticas conservacionistas).

Em virtude de a EUPS utilizar um conjunto de fatores, a sua aplicação gera generalizações, sendo necessários, portanto, discussões e o aprofundamento teórico e metodológico dos fatores que a compõe. Dentre os fatores que mais necessitam de aperfeiçoamento destaca-se o comprimento de rampa (L), não no que tange a sua 
compreensão, mas no seu dimensionamento quando se objetiva estudar grandes áreas, a exemplo de uma bacia hidrográfica. Salienta-se que no presente trabalho os termos rampa, encosta e vertente têm o mesmo significado, ou seja, referem-se ao comprimento horizontal entre a base e o ponto mais alto da encosta.

Quando a EUPS é empregada para a área de uma bacia hidrográfica, se faz necessária uma mensuração e espacialização dos diferentes comprimentos de suas vertentes. Entretanto, os métodos empregados no cálculo de vertente, em sua maioria, contemplam um estado médio por área, o que pode mascarar as oscilações no cômputo de "L" na EUPS. Assim, se fazem necessários métodos que avaliem o comprimento de "L" com mais eficácia e melhor resolução espacial.

Neste contexto, o presente trabalho objetiva apresentar o modelo (RAMPA) capaz de mensurar o comprimento das vertentes em uma bacia hidrográfica, ou seja, o fator (L) inserido na EUPS, a partir dos conceitos matemáticos de limite e derivada, implementado através de uma pseudolinguagem de programação criada no aplicativo "Linguagem Espacial para Geoprocessamento Algébrico - LEGAL", disponível no software SPRING (Sistema de Processamento de Informação Geográfica) desenvolvido pelo INPE (Instituto Nacional de Pesquisas Espaciais).

\section{MATERIAIS E MÉTODOS}

A Microbacia Riacho Passagem possui uma área de $221,7 \mathrm{~km}^{2}$ e uma população de 10.327 habitantes, compreendendo os núcleos urbanos de Itaú e Rodolfo Fernandes. Sua área abrange, ainda, parte da zona rural dos municípios de Severiano Melo e Tabuleiro Grande, na divisa do Estado com o Ceará. Encontra-se inserida na Mesorregião Oeste Potiguar e Microrregião de Pau dos Ferros a uma distância de $380 \mathrm{~km}$ da capital do Estado, Natal (Figura 01). 
Figura 01 - Localização de área de estudo em relação ao Brasil e ao Estado do Rio Grande do Norte.

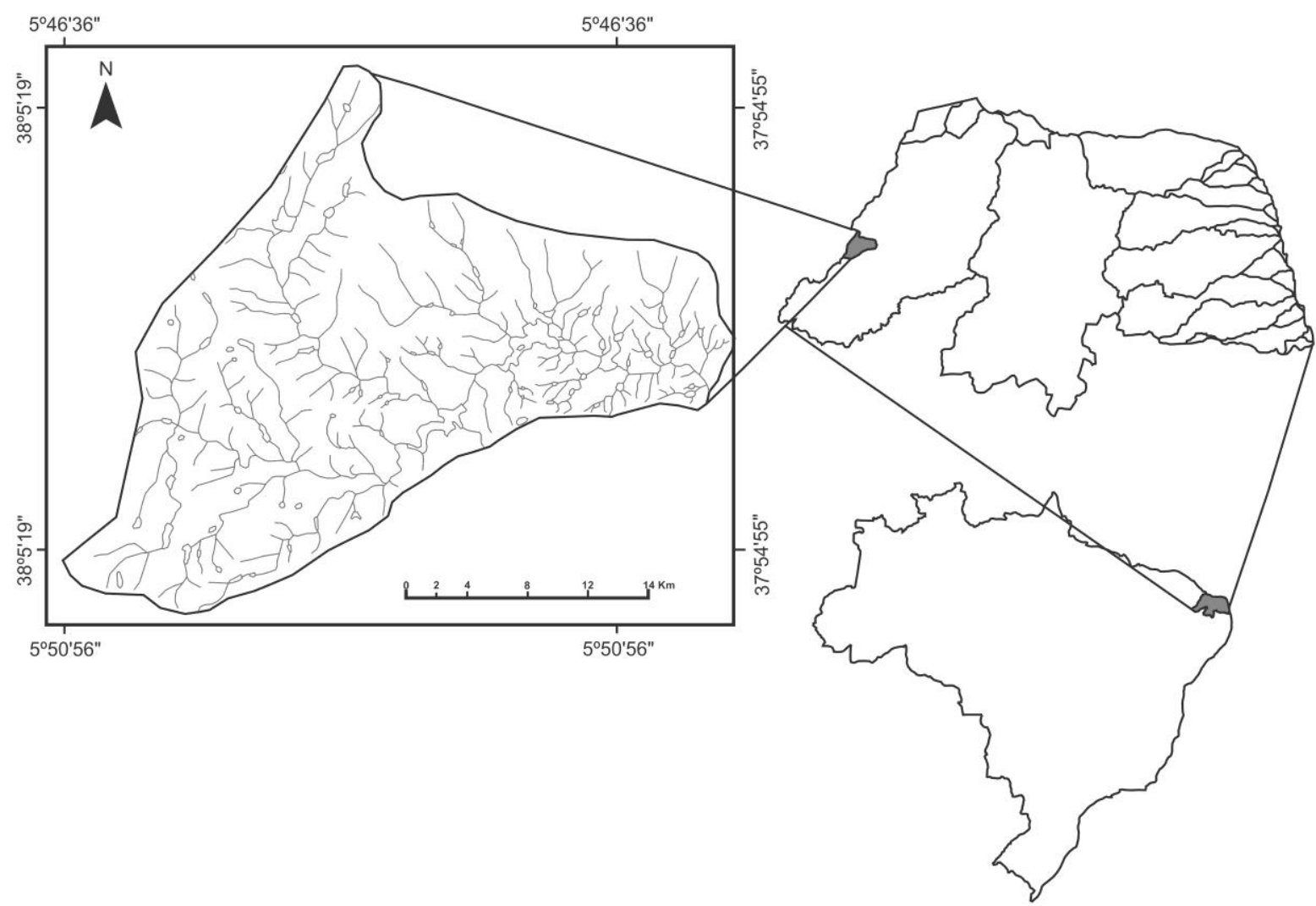

Os dados utilizados foram imagens de radar, em forma de grade numérica do terreno, escala 1:250.000, Sistema geodésico de referência World Global System 1984 (WGS 84), com pixel de $94 \mathrm{~m}$ (resolução espacial), sendo posteriormente interpolado para a resolução espacial de $20 \mathrm{~m}$. A imagem utilizada foi gerada no projeto Shuttle Radar Topography Mission (SRTM), obtidas através de sensores a bordo do ônibus espacial Endeavour, em uma parceria das agências espaciais dos Estados Unidos (NASA e NIMA), Alemanha (DLR) e Itália (ASI) (AMORIM, 2007) e obtidas livremente no site da Empresa Brasileira de Pesquisa Agropecuária (EMBRAPA).

No SPRING o sistema de coordenadas foi alterado para Universal Transversal de Mercator - UTM e o Datum para SAD69, com o objetivo de compatibilizar com as demais informações do Banco de Dados em uso, bem como para possibilitar o cálculo do fator L. Todas as etapas de processamento das informações foram executadas nos softwares SPRING versão 5.1.2 e SURFER versão 8.2. 
Concepção do modelo RAMPA

O método mais simples para o cálculo do comprimento de rampa é o Teorema de Pitágoras, todavia a sua utilização generaliza as variações topográficas ao longo da rampa, ou seja, a mensuração é feita com base nas relações geométricas de um triângulo retângulo. Segundo Christofoletti (1980), o comprimento retilíneo da superfície da vertente (L) corresponde ao segmento da reta que une os pontos superior e inferior de um perfil, desprezando as variações topográficas ao longo da vertente.

Na dedução do modelo proposto (RAMPA), em princípio, considere a linha PST a seção de uma colina situada entre dois vales P e T (Figura 01). A linha PST representará, por exemplo, o perfil longitudinal de um caminho íngreme que galga uma colina.

Sobre a reta 0 e $\mathrm{X}$ estão assinaladas as distâncias em metros (medidas horizontalmente), sobre 0 e Y são medidas as altitudes em metros. Suponhamos que 0 e X estejam ao nível de base local.

Quando se parte da origem $\mathrm{P}$ e se segue o caminho em direção a $\mathrm{S}$, a altura varia à medida que varia a distância, logo a altura é uma função da distância. Dessa forma, a altura é a função, enquanto a distância é a variável, logo a curva PST representa a função "altitude" (Figura 02), tornando deduzível a função "inclinação".

Figura 02 - Perfil longitudinal de uma colina.

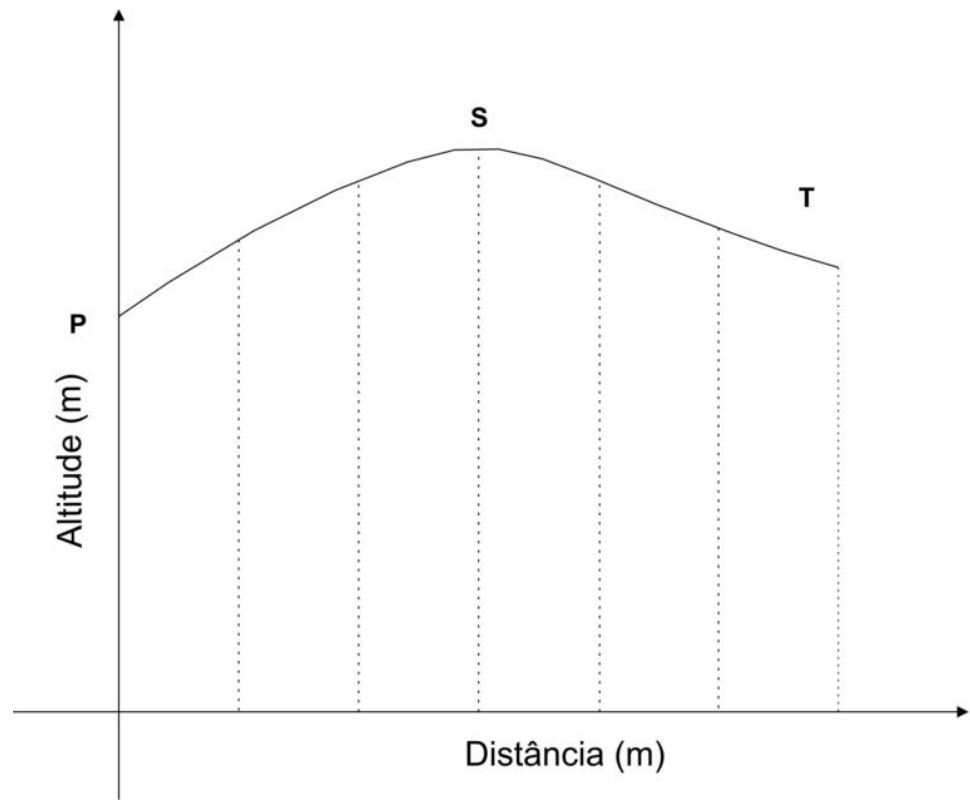


Inicialmente, para não alterar a Figura 2, deslocamos o nosso perfil mudando a origem. Se PST representa a altitude, MN representará a mesma altitude desse nível, por exemplo, um vale.

Suponhamos que exista uma função $\mathrm{f}(\mathrm{x})$ tal que represente o perfil $\mathrm{MN}$, e que $\mathrm{P}_{1}=$ $\left(\mathrm{x}_{1}, \mathrm{y}_{1}\right), \mathrm{P}_{2}=\left(\mathrm{x}_{2}, \mathrm{y}_{2}\right), \ldots \mathrm{P}_{\mathrm{n}}=\left(\mathrm{x}_{\mathrm{n}}, \mathrm{y}_{\mathrm{n}}\right)$ são pontos no gráfico da função $\mathrm{f}(\mathrm{x})$, tal que $\mathrm{y}_{1}=\mathrm{f}\left(\mathrm{x}_{1}\right)$, $\mathrm{y}_{2}=\mathrm{f}\left(\mathrm{x}_{2}\right), \mathrm{y}_{3}=\mathrm{f}\left(\mathrm{x}_{3}\right), \ldots \mathrm{y}_{\mathrm{n}}=\mathrm{f}\left(\mathrm{x}_{\mathrm{n}}\right)$, e que queremos calcular a reta tangente ao gráfico de $\mathrm{f}(\mathrm{x})$ em $\mathrm{p}_{\mathrm{i}}$ (Figura 02). Desde que essa reta tangencie a linha reta que contém o ponto $\mathrm{P}$, tem-se uma aproximação do gráfico de $\mathrm{f}$ nas vizinhanças de $\mathrm{P}$, conforme Figura 3.

Figura 03 - Perfil longitudinal de uma colina em coordenadas cartesianas.

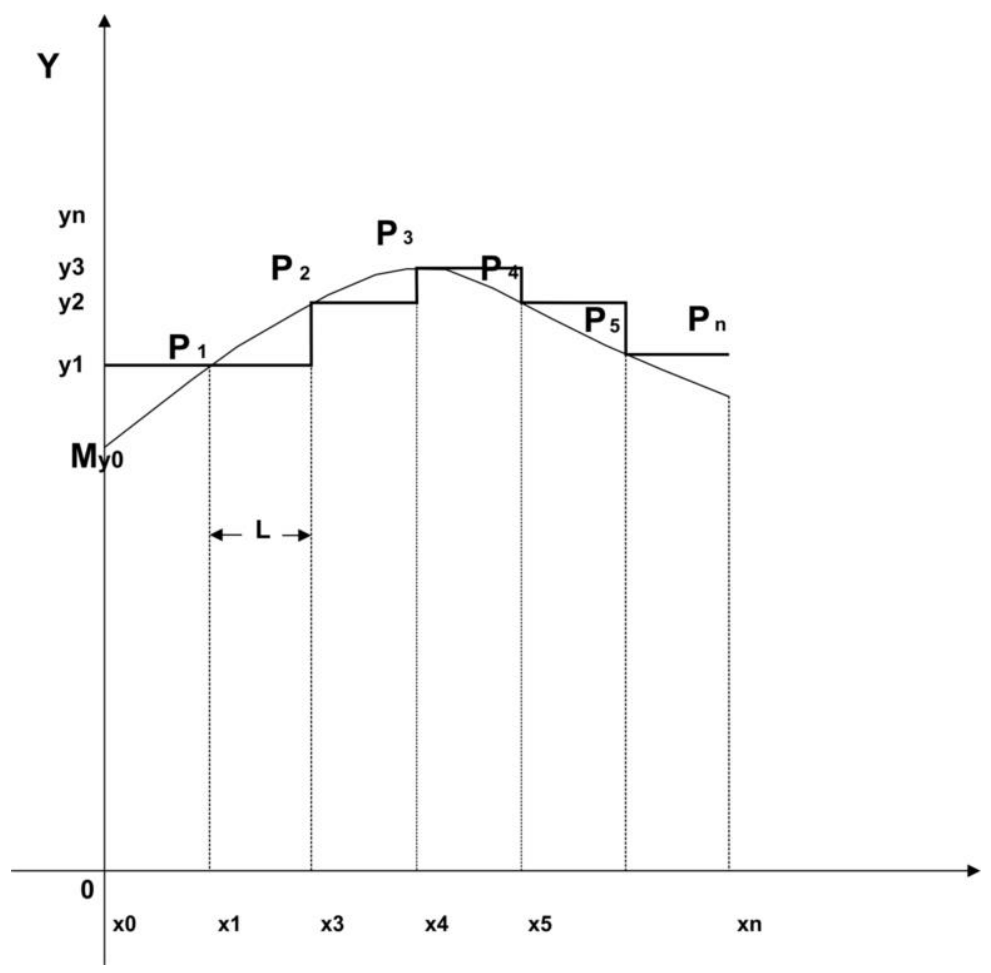

Medimos a inclinação do perfil MN em cada ponto, fazendo a divisão por seguimentos de reta, em primeiro lugar no ponto A. No entanto, suponhamos que precisássemos desenhar essa reta tangente precisamente, já que uma linha reta no plano é completamente determinada quando sabemos o seu coeficiente angular e um ponto pertencente a ela. Assim, só precisamos calcular o coeficiente angular da reta tangente.

A Figura 04 mostra um ponto $\mathrm{P}_{2}$, no gráfico de f, próximo ao ponto $\mathrm{P}_{1}$. $\mathrm{O}$ segmento de reta $\overline{P_{1}} \overline{P_{2}}$ que liga os dois pontos da curva é chamado secante, e a linha reta contendo $\mathrm{P}_{1}$ e 
$\mathrm{P}_{2}$ é chamada reta secante ao gráfico de $\mathrm{f}$. A coordenada $\mathrm{x}$ de $\mathrm{P}_{1}$ é $\mathrm{x}_{1} \mathrm{e}$, se a coordenada $\mathrm{x}$ de $\mathrm{P}_{2}$ difere da coordenada $\mathrm{x}$ de $\mathrm{P}_{1}$ por uma pequena quantidade $\Delta \mathrm{x}$, então a coordenada $\mathrm{x}$ de $\mathrm{P}_{2}$ é $\mathrm{x}_{1}+\Delta \mathrm{x}$.

Figura 04 - Perfil longitudinal de uma colina com discretização da função f(x).

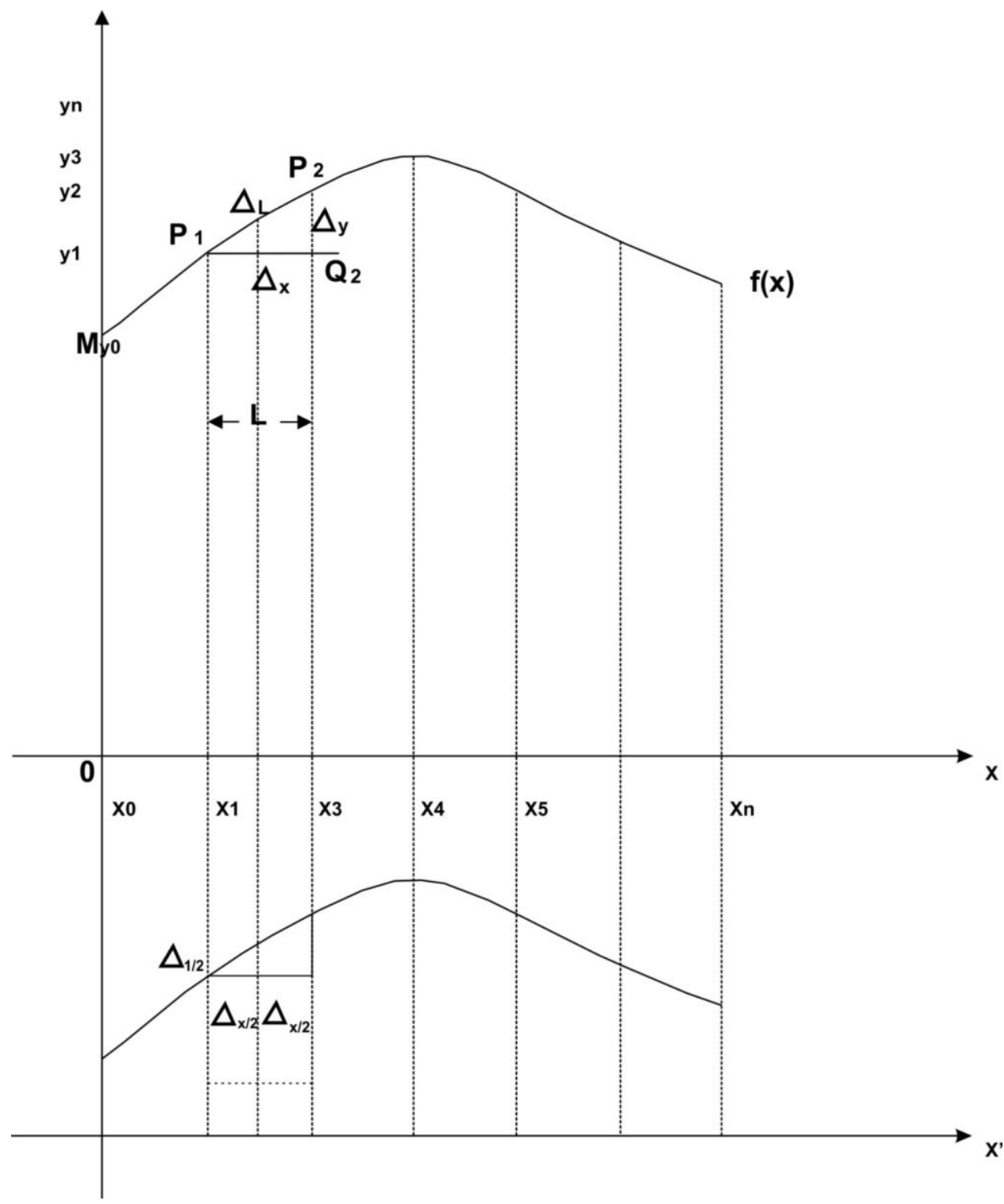

Como $\mathrm{P}_{2}$ pertence ao gráfico de $\mathrm{f}$, segue-se que a coordenada y de $\mathrm{P}_{2}$ é $\mathrm{f}\left(\mathrm{x}_{1}+\Delta \mathrm{x}\right)$. De novo, a coordenada y de $\mathrm{P}_{2}$ difere da coordenada $\mathrm{P}_{1}$ por uma pequena quantidade $\Delta \mathrm{y}$, onde:

$$
\Delta y=f\left(x_{1}+\Delta x\right)-y_{1}=f\left(x_{1}+\Delta x_{1}\right)-f\left(x_{1}\right)
$$


Assim,

$$
\mathrm{P}_{2}=\left(\mathrm{x}_{1}+\Delta \mathrm{x}, \mathrm{f}\left(\mathrm{x}_{1}+\Delta \mathrm{x}\right)\right)=\left(\mathrm{x}_{1}+\Delta \mathrm{x}, \mathrm{y}_{1}+\Delta \mathrm{y}\right)
$$

Pela fórmula do coeficiente angular, a inclinação da secante $\overline{P_{1}} \overline{P_{2}}$ é:

$$
\frac{f\left(x_{1}+\Delta x\right.}{\left(\left(x_{1}+\Delta x\right)-x_{1}\right)}=\frac{\left(f\left(x_{1}+\Delta x\right)-f\left(x_{1}\right)\right)}{\Delta x}=\frac{\Delta y}{\Delta x}
$$

Assim, a reta secante também tem inclinação $\frac{\Delta y}{\Delta x}$.

Agora se fizermos $\Delta x$ tender a zero, o ponto $P_{2}$ se moverá sobre a curva $y=f(x)$ e tenderá ao ponto $\mathrm{P}_{1}$; além disso, a reta secante irá girar em torno do ponto $\mathrm{P}_{1}$ e tenderá, também, para a reta tangente. Assim, enquanto $\Delta \mathrm{x}$ tende a zero, a inclinação $\Delta \mathrm{y} / \Delta \mathrm{x}$ da reta secante tende para a inclinação da reta tangente. Logo, a reta secante " $\Delta \mathrm{L}$ " com inclinação dada por $\Delta \mathrm{y} / \Delta \mathrm{x}$, coincide com a linha reta no plano xy contendo o ponto $\left(\mathrm{x}_{1}, \mathrm{y}_{1}\right)$ e tendo como coeficiente angular "a" dada por:

$$
\mathrm{a}=\operatorname{tg}(\theta)=\Delta \mathrm{y} / \Delta \mathrm{x}
$$

Suponhamos que o ponto $\mathrm{P}_{1}$ esteja na metade da reta tangente do triângulo (Figura 03), logo a base do triângulo será $\Delta \mathrm{x} / 2$, e a inclinação (reta de tangência da vertente) será $\Delta \mathrm{L} / 2$. Observe que $\Delta \mathrm{y}$ depende de $\Delta \mathrm{x}$, visto que, quanto menor for $\Delta \mathrm{x}$, mais próximo $\Delta \mathrm{y}$ estará de dy, o que permite considerar, fazendo uso de derivadas direcionais, e por analogia, nas direções x e y:

$$
\Delta \mathrm{y} \approx \partial \mathrm{y} \text { e } \Delta \mathrm{x} \approx \partial \mathrm{x}
$$

No novo triângulo temos o valor de sua base, " $\partial \mathrm{x}$ " e do ângulo " $\theta$ ", e pelos conceitos da trigonometria teremos:

$$
\begin{aligned}
& \partial \mathrm{L}_{\mathrm{x}}=\cos ^{-1}(\theta) \partial \mathrm{x} \\
& \partial \mathrm{L}_{\mathrm{y}}=\cos ^{-1}(\theta) \partial \mathrm{y}
\end{aligned}
$$

Logo no sistema de equações envolvendo o comprimento de rampa (L), a resolução espacial da imagem (x) ou (y) e a inclinação da vertente $(\theta)$. Além de representar as variações 
no relevo, o sistema de equações permite determinar aproximadamente variações do comprimento da rampa a cada pixel.

Na implementação do modelo "RAMPA" utilizou-se da Linguagem Espacial para Geoprocessamento Algébrico (LEGAL), que é um aplicativo disponível no SPRING e permite ao usuário implementar as funções desejadas. Para Câmara (1995) o objetivo do LEGAL é prover um ambiente que possibilite infinitas aplicações em análise geográfica, incluindo operação sobre geocampos e geo-objetos.

\section{RESULTADOS E DISCUSSÃO}

Cada vez mais cresce a necessidade de técnicas capazes de mensurar as características do meio natural, e que sua manipulação ocorra de forma ágil, rápida e precisa. Nesse sentido, as técnicas disponíveis nos programas de geoprocessamento estão se consolidando como as mais empregadas quando se busca conhecer e estudar as condições da superfície terrestre. Porém, é preciso o desenvolvimento de modelos, que poderão posteriormente ser inseridos como ferramentas para melhor atender as especificidades da natureza dos trabalhos na área da Geografia Física.

O modelo proposto no presente artigo possibilita melhorar de modo significativo os estudos voltados à erosão do solo, onde é necessário definir o comprimento de rampa em uma área qualquer, por exemplo, uma bacia hidrográfica. O termo rampa é utilizado na Equação Universal de Perda de Solos (EUPS) para se referir ao comprimento de vertente e, aqui, representa o foco principal do trabalho.

A figura 05 apresenta a espacialização dos comprimentos de rampa na Microbacia Riacho Passagem. Saliente-se que as variações verticais que se observam não correspondem à elevação no relevo da área de estudo (rugosidade da figura), mas as variações representam as áreas com maiores comprimentos de rampa, o que não necessariamente corresponde às áreas com maiores variações topográficas, mesmo porque as maiores vertentes são encontradas nas áreas mais planas da microbacia.

Conforme destacado no mapa (Figura 5), os maiores comprimentos de rampa se encontram na porção leste junto à desembocadura da microbacia. Os resultados encontrados pelo modelo RAMPA foram avaliados por checagem em campo em toda a microbacia, utilizando um GPS de navegação da marca Garmim. Para tanto, foram comparados os valores gerados pelo modelo e o tamanho das rampas em campo, sem considerar a declividade. 
Figura 05 - Espacialização do comprimento de rampa na Microbacia Riacho Passagem/RN.

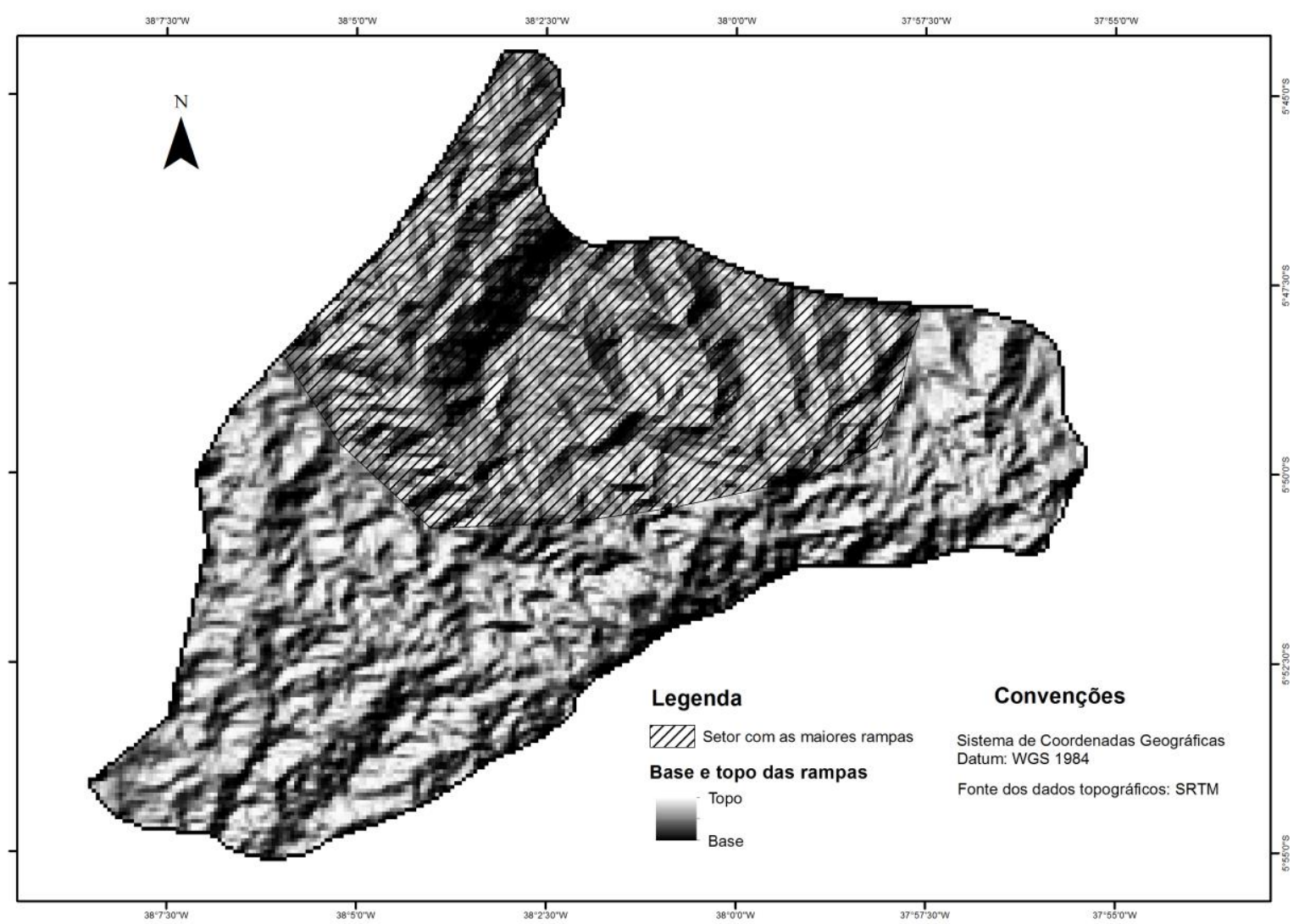

As verificações a campo comprovaram a eficiência do modelo para o cálculo de rampa, uma vez que foram encontrados, na área com rachurada (Figura 05), os maiores comprimentos de rampa, como pode ser observado na Figura 6. Nessa área predomina um relevo variando de plano a suavemente ondulado, com vertentes convexas e solo variando de LATOSSOLOS para NEOSSOLOS REGOLITICOS. 
Figura 6 - Cenário da região na microbacia onde se concentram os maiores comprimentos de rampa. Área desnudada com 300m de comprimento no qual a seta indica o sentido do escoamento superficial.

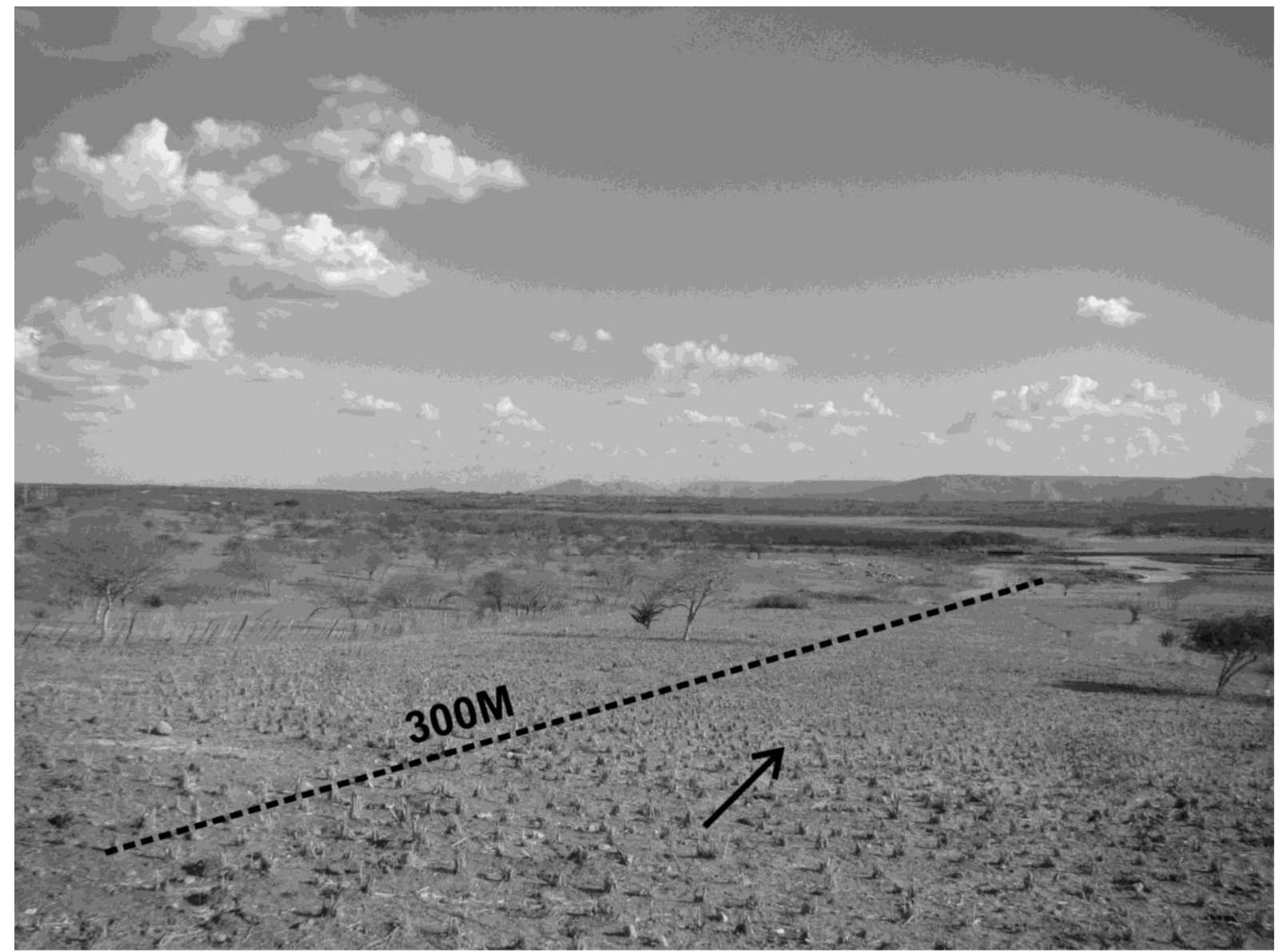

Fonte: $\mathrm{O}$ autor, janeiro de 2008.

Os resultados encontrados no trabalho se mostram bastantes significativos, uma vez que proporcionam um ganho científico de custo-benefício, em virtude de serem obtidos de forma automática e espacializada, possibilitando a avaliação por área, o que torna o mapa mais sensível ao real comportamento dos valores de (L).

O método de cálculo do comprimento de rampa e os resultados encontrados no presente trabalho possibilitam aperfeiçoamentos nos métodos que objetivam mensuração de perda de solo por técnicas de geoprocessamento. Esses resultados são diferentes de Costa (2005), que utilizou um valor médio para cada subunidade trabalhada. Ela determinou o comprimento de rampa considerando o parâmetro da extensão do percurso superficial como sendo igual ao comprimento médio das vertentes em cada unidade de análise. Ou seja, uma generalização do comprimento de vertente.

O método que mais se aproxima do proposto, é o de Silva (2003), implementado a partir do algoritmo desenvolvido por Desmet \& Govers para calcular o comprimento de 
rampa utilizando como base uma grade retangular. $\mathrm{O}$ algoritmo funciona calculando a declividade, a direção de fluxo e a quantidade de fluxo que se acumulou a montante de um determinado pixel.

Assim, se faz necessária a aplicação dos três métodos: Costa (2005), Silva (2003) e o modelo proposto no presente trabalho, a partir de um estudo experimental, para avaliar os dados gerados nos modelos, aplicando-os na EUPS e checando os seus resultados com os dados coletados em campo. Essa comparação terá como base um experimento onde serão medidos os comprimentos de rampa utilizando um GPS geodésico e a perda de solo através de uma parcela, considerando apenas a influência do comprimento de rampa na erosão do solo.

\section{CONSIDERAÇÕES FINAIS}

A rapidez atualmente exigida no diagnóstico do potencial à degradação do solo estabelece uma maior agilidade e precisão dos modelos envolvidos nesse pleito. Para tanto, o modelo proposto se aplica perfeitamente aos estudos de predição de perda de solo em bacias hidrográficas, quando se objetiva tratar as informações de forma espacial utilizando as técnicas de geoprocessamento em ambiente SIG. Todavia, outras aplicações poderão ser vislumbradas, tais como estudos geomorfológicos e fitogeográficos.

O modelo poderá servir de base para o fornecimento da variável rampa da Equação Universal de Perda do Solo (EUPS), uma vez que ela utiliza o comprimento das vertentes (rampa) na estimativa de perda de solo. As áreas mais íngremes, com maiores comprimentos de rampa, facilitam a erosão dos solos, na medida em que aumentam a velocidade do escoamento superficial, com perdas de sedimentos de modo exponencial. Esse tipo de informação é indispensável no planejamento e gestão ambiental de bacias hidrográficas e pode ser trabalhado em um SIG e relacionado com outras informações de cunho hidrológico.

$\mathrm{Na}$ análise de relevos, o modelo para cálculo de rampa poderá ser utilizado nos estudos de topossequências, permitindo calcular os comprimentos de vertentes em toda uma bacia hidrográfica, informação que possibilita a elaboração de perfis de forma espacializada. Tal como a utilização em estudos morfométricos, onde é possível determinar os comprimentos de rampas predizendo a ocorrência de um determinado tipo de relevo, características do escoamento superficial e processos geomorfológicos atuantes. 
O modelo RAMPA possibilitou detalhar os comprimentos das encostas, de forma espacial na microbacia, quando normalmente esse dado só é obtido com levantamentos de campo. Para uma aplicação prática, esses dados poderão nortear a efetivação de métodos de controle da erosão laminar, como a construção de linhas de seixos perpendicular ao sentido do escoamento nas vertentes e barramentos com blocos de rochas nos canais de primeira ordem.

Os resultados aqui apresentados ainda são preliminares e, portanto, deverão passar por novos testes onde serão realizadas medições de campo utilizando um GPS geodésico, o que proporcionará maior precisão nos dados trabalhados, bem como a comparação com outras fontes de dados topográficos, por exemplo: curvas de nível de cartas topográficas e imagens topográficas disponíveis, de forma a balizar a consistência do modelo.

\section{REFERÊNCIAS}

AMORIM, Rodrigo de Freitas. Potencial natural à erosão (PNE) na Microbacia do Riacho Passagem Itaú/RN. 66 f. Monografia (Bacharelado em Geografia) - UFRN. CCHLA, Dep. De Geografia, Natal/RN, 2007.

BERTONI J.; LOMBARDI Neto. Conservação do solo. 4. ed. São Paulo: Ícone, 1999. 355 p. BERTRAND, G. Paisagem e geografia física global: esboço metodológico. Caderno de Ciências da Terra. Tradução O. Curz. São Paulo, v. 13, USP, 1972. 27 p.

CÂMARA, G. Modelos, Linguagens e Arquiteturas para Bancos de Dados Geográficos. Tese de Doutoramento em Computação Aplicada. São José dos

Campos, INPE, 1995.

CHRISTOFOLETTI, Antônio. Geomorfologia. 2. ed. São Paulo: Edgar Blucher, 1980. 188p.

CHRISTOFOLETTI, Antônio. Modelagem de sistemas ambientais. São Paulo: Edgar Blucher, 1999. 236p.

COSTA Ana Lúcia Carneiro da. Estudo da vulnerabilidade à erosão com a aplicação da Equação Universal de Perda de Solo na Alta Bacia Hidrográfica do Rio Jacaré Pepira, utilizando SIG/SPRING. 168 f. (Dissertação de Mestrado) - Programa de Pós Graduação em Geociências, Rio Claro/SP, 2005.

SILVA, Valtercides Cavalcante. Cálculo automático do fator topográfico (ls) da EUPS, na Bacia do Rio Paracatu. Pesquisa Agropecuária Tropical; n 33, p. 29-34, 2003.

SPRING versão 5.1.2. São José dos Campus/SP: Instituto Nacional de Pesquisas espaciais, 2009. Disponível em: <http//:www.dpi.inpe.br/spring> Acesso em 06 ago. 2009. 
EMPRESA BRASILEIRA DE PESQUISA AGROPECUÁRIA - EMBRAPA. Relevo brasileiro. Disponível em: http://www.relevobr.cnpm.embrapa.br. Acesso em: 22 set. 2009.

WISCHMEIER, W. H.; SMITH, D. D. Predicting rainfall erosion losses: a guide planning. Washington, D. C. USDA,1978. (Handbook, 537). 